\title{
Adaptive Parameter Identification of a Fuel Cell System for Health-Conscious Energy Management Applications
}

\author{
Mohsen Kandidayeni, Student Member, IEEE, Hicham Chaoui, Senior Member, IEEE, Loïc Boulon, \\ Senior Member, IEEE, and João Pedro F. Trovão, Senior Member, IEEE
}

\begin{abstract}
Since a proton exchange membrane (PEM) fuel cell (FC) has time-varying characteristics, its online characteristics estimation (voltage, power, internal resistance, etc.) is becoming a key step in designing an energy management strategy (EMS) for hybrid FC vehicles. In this respect, this paper proposes a new method based on Lyapunov adaptation law to estimate the linear and nonlinear parameters of a renowned PEMFC model in the literature. Unlike most of similar estimators, the suggested approach determines the maximum current, which is a nonlinear parameter, online while guaranteeing the system closed-loop stability. This parameter is normally assumed to be constant while it changes through time owing to degradation and operating conditions variation. This alteration makes the model imprecise while extracting some important characteristics, such as maximum power and polarization curve. Therefore, it needs to be regularly updated along with other parameters. To demonstrate the capability of the suggested method, a detailed comparison is provided with the well-known extended Kalman filter (EKF) as an attested nonlinear estimator. Moreover, to highlight the effectiveness of the nonlinearity consideration, a comparison with $\mathrm{KF}$ is performed where the nonlinear parameter is considered constant. The performed experiments on a 500-W PEMFC show that the proposed method can be over twice as accurate as EKF and KF concerning the estimation of maximum power and current while its runtime is nearly half of them.
\end{abstract}

Index Terms-Kalman filter, health assessment, Lyapunov stability, online modeling, fuel cell

\section{INTRODUCTION}

\section{A. General context}

$\mathrm{H}$ YBRID fuel cell (FC) vehicles are considered as one the most promising technical solutions in the battle to confront the climate change crisis [1]. The performance of this multiple energy source system highly depends on the design of an

This research was funded by Fonds de Recherche du Québec-Nature et technologies (FRQNT) [Bourses de recherche postdoctorale B3X, file number: 284914], Natural Sciences and Engineering Research Council of Canada (NSERC) [RGPIN-2018-06527 and RGPIN-2017-05924], and Canada Research Chairs program [950-230863 and 950-230672].

M. Kandidayeni is with e-TESC and IRH labs, Department of Electrical Engineering and Computer Engineering, University of Sherbrooke, Sherbrooke, QC, J1K 2R1, Canada (email: mohsen.kandidayeni@usherbrroke.ca).

H. Chaoui is with the Intelligent Robotic and Energy Systems (IRES) Research Group, Department of Electronics, Carleton University, Ottawa, ON, Canada (email: hicham.chaoui@carleton.ca) appropriate energy management strategy (EMS) [2, 3]. Thus far, numerous EMSs have been proposed for these vehicles which are normally based on proton exchange membrane FC (PEMFC) models with static characteristics (voltage and power) [4]. For instance, in [5], an EMS is suggested for a $\mathrm{FC} /$ battery hybrid powertrain using model predictive control (MPC). The PEMFC is modeled by a quadratic fitted hydrogenvs-power equation. In [6], Pontryagin's minimum principle (PMP) is utilized to distribute the power between the FC and battery while considering the durability of the sources. The FC model is based on the experimental measurements, and the costate is updated online according to the battery SOC. In [7], an EMS based on adaptive droop control combined with a multimode strategy and an equivalent consumption minimization strategy (ECMS) is proposed for a multiple power source system. The PEMFC model is based on polarization curve behavior and the maximum and minimum FC power points are among the considered constraints. In [8], fuzzy logic control (FLC) and adaptive control theory are combined to develop an EMS. The authors recommend revising the values of FLC membership functions after a while since the PEMFC voltage declines through time owing to degradation. Moreover, in [9], a quadratic programming (QP) based strategy is proposed for a FC-battery vehicle in which the authors indicate that not updating the constraints of an EMS, such as PEMFC power and efficiency values, can increase the hydrogen consumption up to $6.6 \%$ for the studied cases.

These reflections illustrate that considering the FC state of health $(\mathrm{SOH})$ while developing an EMS seems essential as the energetic performance of the FC varies through time owing to different uncertainties, and this can result in the mismanagement of the vehicle [10]. Consequently, some creative endeavors have been made to augment the health awareness of EMSs.

L. Boulon, is with Hydrogen Research Institute, Electrical and Computer Engineering Department, Université du Québec à Trois-Rivières, QC, Canada (email: loic.boulon@uqtr.ca).

S. Kelouwani is with the Hydrogen Research Institute, Department of Mechanical Engineering, Université du Québec à Trois-Rivières, QC G9A 5H7, Canada (e-mail: sousso.kelouwani@uqtr.ca).

J. P. F. Trovão is with the Department of Electrical Engineering and Computer Engineering, University of Sherbrooke, Sherbrooke, QC, J1K 2R1, Canada (email: Joao.Trovao@USherbrooke.ca). 


\section{B. Literature study}

Several papers have focused on the inclusion of a degradation model to develop a decision-making strategy based on the health state of the power sources [11-14]. To take some examples, in [11], a PEMFC degradation model based on high load, low load, and load changes has been combined with a MPC. In [12], particle filter is used to estimate the lifetime of the FC, and a FLC based EMS is accordingly developed. In [13], a degradation model is proposed to extract the polarization and efficiency curves of the PEMFC in different health states, and it is then combined with an optimization based EMS. Although the discussed papers have provided valuable solutions, modeling the degradation mechanism under automotive conditions is a difficult task and is still an open problem in the literature. Moreover, the change of operating conditions that are not included in the model can also impact the FC maximum efficiency (ME) and maximum power (MP) that are considered as the most used constraints while developing an EMS.

To avoid the mentioned complications, the use of an extremum seeking technique based on perturb and observe has been practiced in some papers [15-17]. For example, in [15], a fractional-order method based on gradient optimization process is proposed to track the extremum value of a static nonlinear FC system. In [16], a multi-objective optimization problem based on extremum seeking is introduced to meet the required levels of voltage regulation, ME, and MP. The perturb and observe based methods are normally desirable due to their convenient deployment in an EMS design. Nevertheless, once the concurrent identification of several operating points is in demand, like in an online EMS, the complexity of these algorithms also increases. There are also some other techniques, such as hierarchical technique (multi-objective optimization and data-driven constraint predictive control) [18], sliding mode variable structure [19], and systemic management of FC current and temperature in [20], to provide efficient and stable operation of the FC system. While these methods have shown robust and satisfactory results, they are dependent on a wide range of experimental maps to be used as an optimal reference.

To evade the above-reviewed issues, a new paradigm has been introduced for formulating a health-aware EMS based on adaptive parameter identification [21,22]. The foundation of this concept is presented in Fig. 1 through a three-step process.
In the first step, the parameters of a FC semi-empirical model are updated by an estimator while the vehicle is under operation. In the second step, the required PEMFC characteristics for updating the constraints of the EMS are extracted from the updated model and forwarded to the strategy to define reliable reference signals in the final step. Recursive least square (RLS) is utilized to identify the parameters of a polynomial function representing efficiency-vs.-power in [23] and hydrogen consumption-vs.-power in [24]. In [25], adaptive RLS is used to extract the ME and MP points of a FC system in an electric tram based on which the safe operating zone for the proposed EMS is updated. In [26], forgetting factor RLS combined with sequential QP is used to identify the maximum efficiency range for an ECMS. In [27], Ettihir et al. have used RLS to extract the parameters of a current-dependent FC model, suggested by Squadrito et al. [28], and in [29], unscented Kalman filter (KF) is used for updating the same model. However, they have not commented on the distinctive aspects of the methods. In [30], different categories of PEMFC models and identification algorithms are investigated for EMS application, and the performance of Squadrito et al. model is compared with the one suggested by Amphlett et al. [31, 32] using RLS and KF. The obtained results indicate that the multiinput model (Amphlett) is more precise than the single-input one (Squadrito), and KF is slightly more accurate than RLS. Although the discussed adaptative filters have been able to estimate the parameters of a PEMFC system, they all lack stability analysis which is necessary to validate the dependability in the long term. In [33], an adaptive identification method based on Lyapunov is proposed to estimate two parameters of a nonlinear model which only considers activation and ohmic losses.

\section{Contributions and organization}

This paper focuses on the first and second steps of the proposed concept in Fig. 1. In this regard, a Lyapunov based adaptation law is proposed to estimate the linear and nonlinear parameters of a renowned semi-empirical model, proposed by Amphlett et al, while guaranteeing the system stability. Then, as an example, some important PEMFC characteristics (state of power, voltage, and internal resistance) are extracted which can be used in the formulation of an EMS in future. The targeted nonlinear parameter for estimation is the PEMFC maximum

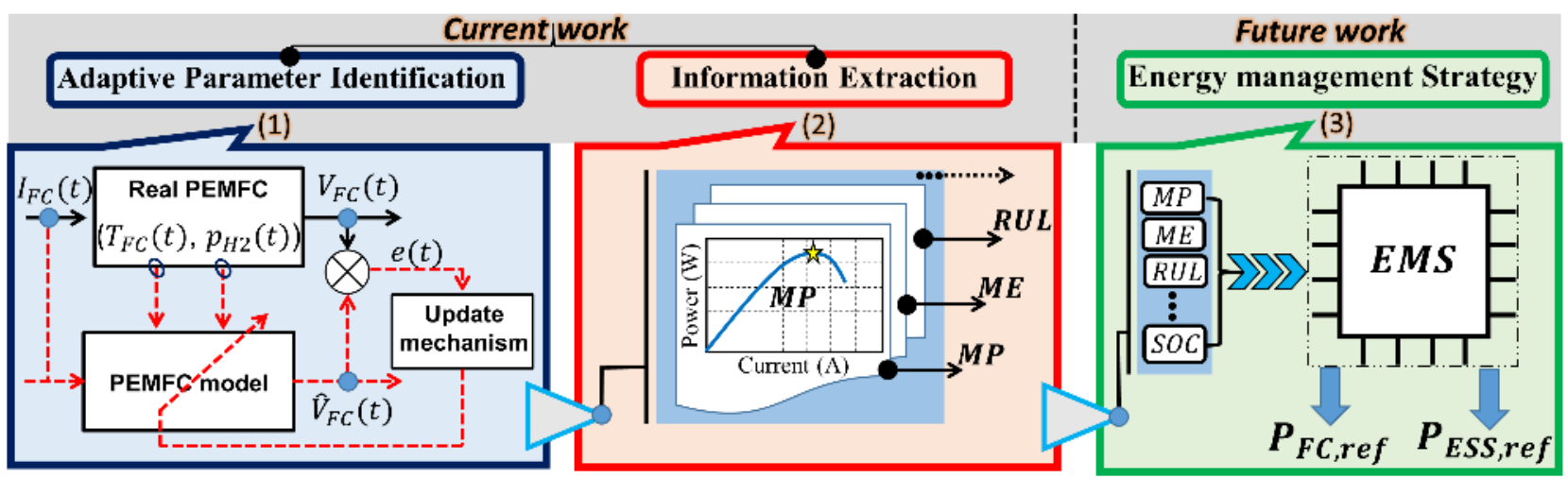

Fig. 1. General structure for integrating a FC adaptive parameter identification into an EMS design. $\left(P_{F C, r e f}\right.$ denotes the requested power from $\mathrm{FC}$ and $P_{E S S, r e f}$ is the one from energy storage system) 
current which is prone to changes owing to uncertainties caused by operating conditions variation and degradation. This parameter is highly sensitive concerning the estimation of voltage, as discussed in [34], and its variation can make the PEMFC model inaccurate. However, it is either assumed to be constant in other similar papers or estimated offline using metaheuristic algorithms [35, 36]. Extended KF has been used to estimate this parameter online in [30]. However, it lacks stability proof, and has higher computational burden compared to the proposed adaptive method. Compared to the performed identification in [33] which does not consider concentration region and has only two parameters for estimation, in this work, three losses (activation, ohmic, and concentration) are taken into account, and the estimator identifies online nine parameters. To experimentally validate the performance of the proposed nonlinear adaptive parameter estimator (NLAPE), the characteristics of a 500-W open cathode FC are estimated by updating the parameters of the semi-empirical model. Furthermore, the put forward method is compared with EKF, as a reliable nonlinear estimator, to be validated in accuracy terms. It is also compared with KF to show the necessity of updating the nonlinear parameter.

Section II describes the model of the PEMFC. The proposed NLAPE is detailed in section III. Section IV details the experimental set-up and the obtained results are discussed in section V. Finally, the conclusion is given in section VI.

\section{Fuel Cell Modeling}

Polarization curve analysis is suitable for demonstrating the impact of operating parameters, such as temperature, flow rate, relative humidity, power, and efficiency. Fig. 2 illustrates a standard polarization curve, which comprises standard reversible potential and three irreversible losses, namely activation, ohmic, and concentration. The first loss is related to the sluggish kinetics of oxygen reduction reaction happening in the low current region. The second one is associated with the resistance to ions flow in the electrolyte and electrons flow through the electrode. The third one, known as mass transport effect, is due to the restriction of the reactant gas transport across the gas diffusion and electrocatalyst layers and occurs at high current zone.

Among the existing models, the one introduced by Amphlett et al. [31, 32], has been successfully employed for modeling different PEMFC stacks, as discussed earlier. This model has

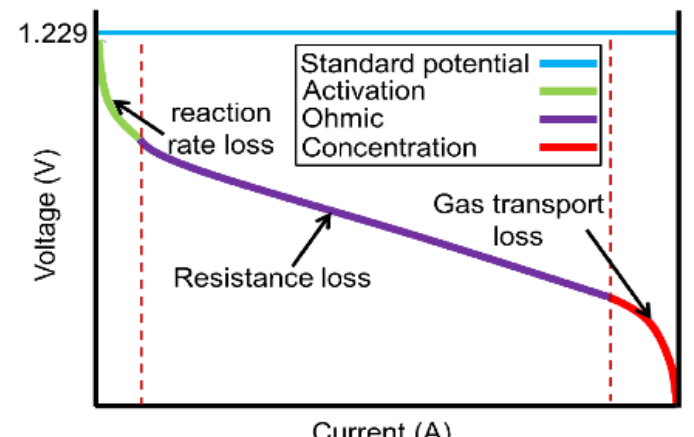

Current $(A)$

Fig. 2. Standard polarization curve of a PEMFC cell. also come under scrutiny in different comparative studies [30, 37]. Although PEMFC technology has been substantially upgraded since this model was introduced, its structure is still appropriate. However, new parameterization techniques are obviously required to fit this model to the performance of a PEMFC. Thus, this model is used in this paper to estimate the voltage of an open cathode PEMFC system and the proposed adaptive estimation method is utilized for its parameterization.

The voltage of the PEMFC stack $\left(V_{F C}\right)$ is given by:

$\left\{V_{F C}=N_{\text {cell }} V_{F C N}\right.$

$\left\{V_{F C N}=E_{\text {Nernst }}+V_{\text {act }}+V_{\text {ohmic }}+V_{\text {con }}\right.$

where $N_{\text {cell }}$ is the number of cells, $V_{F C N}$ is the cell voltage (V), $E_{\text {Nernst }}$ is the reversible cell potential $(\mathrm{V}), V_{\text {act }}$ is the activation loss $(\mathrm{V}), V_{\text {ohmic }}$ is the ohmic loss $(\mathrm{V})$, and $V_{\text {con }}$ is the concentration loss (V). $E_{\text {Nernst }}$ is calculated by:

$E_{\text {Nernst }}=1.229-0.85 \times 10^{-3}\left(T_{F C}-298.15\right)+4.3085 \times$
$10^{-5} T_{F C}\left[\ln \left(P_{H 2}\right)+0.5 \ln \left(P_{O 2}\right)\right]$

where $T_{F C}$ is the stack temperature $(\mathrm{K}), P_{H 2}$ is the hydrogen partial pressure in anode side $\left(\mathrm{N} \mathrm{m}^{-2}\right)$, and $P_{O 2}$ is the oxygen partial pressure in the cathode side $\left(\mathrm{N} \mathrm{m}^{-2}\right)$. $V_{\text {act }}$ is determined by:

$\left\{\begin{array}{l}V_{a c t}=\xi_{1}+\xi_{2} T_{F C}+\xi_{3} T_{F C} \ln \left(C_{O 2}\right)+\xi_{4} T_{F C} \ln \left(I_{F C}\right) \\ C_{O 2}=\frac{P_{O 2}}{5.08 \times 10^{6} \exp \left(-498 / T_{F C}\right)}\end{array}\right.$

where $\xi_{n}(n=1 \ldots 4)$ are the semi-empirical coefficients based on fluid mechanics, thermodynamics, and electrochemistry, $C_{O 2}$ is the oxygen concentration $\left(\mathrm{mol} \mathrm{cm}^{-3}\right)$, and $I_{F C}$ is the PEMFC operating current (A). $V_{\text {ohmic }}$ is given by:

$V_{\text {ohmic }}=-I_{F C} R_{\text {internal }}=-I_{F C}\left(\zeta_{1}+\zeta_{2} T_{F C}+\zeta_{3} I_{F C}\right)$

where $R_{\text {internal }}$ is the internal resistor $(\Omega)$, and $\zeta_{n}(n=1 \ldots 3)$ are the parametric coefficients. Internal resistance is one of the most used degradation indexes as the degradation is generally defined by the increase of the internal resistance leading to a power/voltage decrease in a PEMFC [38]. Finally, $V_{\text {con }}$ is calculated by:

$V_{\text {con }}=B \ln \left(1-\frac{I_{F C}}{I_{\max }}\right)$

where $B$ is a parametric coefficient $(\mathrm{V})$, and $I_{\max }$ is the maximum current $(A)$.

Table I shows the parameters which need to be estimated online by the adaptive estimator. The ranges of these parameters are mostly adopted from [30]. In this study, the calibrated parameters of the PEMFC may slightly differ from the reported values. It is since these parameters vary through time because of the uncertainties arisen from variation of operating conditions and the health state of the PEMFC system. 
TABLE I

INTENDED PARAMETERS FOR IDENTIFICATION [30]

\begin{tabular}{|c|c|c|c|}
\hline \multirow{2}{*}{ Parameter } & \multicolumn{2}{|c|}{ Range } & \multirow{2}{*}{ Unit } \\
\hline & Minimum & Maximum & \\
\hline$\xi_{1}$ & -1.997 & -0.8532 & - \\
\hline$\xi_{2}$ & 0.001 & 0.005 & - \\
\hline$\xi_{3}$ & $3.6 \times 10^{-5}$ & $9.8 \times 10^{-5}$ & - \\
\hline$\xi_{4}$ & $-2.6 \times 10^{-4}$ & $-0.954 \times 10^{-4}$ & - \\
\hline$B$ & 0.0135 & 0.5 & $\mathrm{~V}$ \\
\hline \multirow{2}{*}{$\begin{array}{c}R_{\text {internal }}=f\left(\zeta_{1}, \zeta_{2}, \zeta_{3}\right) \\
I_{\max }\end{array}$} & 0.16 & 0.22 & $\Omega$ \\
\hline & 20 & 42 & A \\
\hline
\end{tabular}

\section{NONLINEAR ADAPTIVE PARAMETER ESTIMATOR}

As reviewed in Section I, several papers have employed recursive filters to estimate the parameters of a FC model [15, $17,22,35]$. While they have achieved satisfactory results, one of the most fundamental concerns is that the stability of these methods can vanish due to the improper initialization or other divergence phenomena. For example, if the covariance matrix does not become nonnegative-definite, KF/EKF can become unstable (divergence phenomenon) [36]. Consequently, the necessity of a stability-based technique using Lyapunov theory has been justified for estimating the parameters of electrochemical devices in many papers [37-39]. Stability proof draws up guidelines on the choice of control/estimation and adaptation laws along with the range of gains where the closedloop system is stable, and it is unique for a given estimator. Lyapunov is a recognized stability theory and has been used for online parameter estimation in different fields [40, 41]. Hence, in this section, an online NLAPE is designed using Lyapunov theory to estimate in real-time the FC parameters. According to (1)-(5), the PEMFC model is a nonlinear system which can be written as,

$y=f(x, u, \Theta)$

where $y$ is the system output, $x$ is the system states, $u$ is the system inputs, and $\Theta$ denotes the unknown parameters for estimation. Looking back at (1)-(5), it is observed that all the parameters are linear considering their functions except for one which is the maximum current $\left(I_{\max }\right)$. In fact, $I_{\max }$ is placed in the nonlinear function. Thus, it needs to be extracted by Taylor series expansion to be able to linearly reformulate the system equation. Applying the Taylor series expansion to (6) leads to,

$y=f(x, u, \widehat{\Theta})+\sum_{i=1}^{n} \tilde{\theta}_{i} \frac{\partial f(x, u, \widehat{\Theta})}{\partial \theta_{i}}+\xi(\tilde{\Theta})$

where $\hat{\Theta}$ is the estimated parameter vector, $\tilde{\Theta}=\Theta-\hat{\Theta}$ is the parameter estimation error vector, and $\xi(\tilde{\Theta})$ is the residual error resulted from higher order terms. For $\Theta$ sufficiently close to $\hat{\Theta}$, these higher order terms of Taylor series expansion will be very close to zero, and so can be dropped to obtain the approximation.

Using (1)-(5), the nonlinear model can be described as,

$V_{F C}=\psi_{1} \theta_{1}+\psi_{2} \theta_{2}+\cdots+\psi_{9} \theta_{9}=\Psi^{T} \Theta$ where, $\Psi$ is a vector of known functions (regressor) obtained by applying (7) to system equation.

$\Psi \in \mathbb{R}^{9}=$

$\left[1, T_{F C}, T_{F C} \ln \left(C O_{2}\right), T_{F C} \ln \left(I_{F C}\right),-I_{F C},-I_{F C} T_{F C},-I_{F C}{ }^{2}, \ln (1-\right.$

$\left.\left.\theta_{9} I_{F C}\right),-I_{F C} \theta_{8} /\left(1-\theta_{9} I_{F C}\right)\right]$

$\Theta \in \mathbb{R}^{9}$ is a vector of unknown parameters, as listed in Table I. Therefore, the FC voltage estimation law is defined as,

$\widehat{V}_{F C}^{r}=\psi_{1} \hat{\theta}_{1}+\psi_{2} \hat{\theta}_{2}+\cdots+\psi_{9} \hat{\theta}_{9}=\Psi^{T} \hat{\Theta}$

where the hat accent placed on top of each variable ", ,", represents the estimate of that variable, and

$\widehat{V}_{F C}^{r}=\widehat{V}_{F C}-K_{p} e-K_{i} \int e$

where, $K_{p}$ and $K_{i}$ are the proportional and integral gains, respectively, and $e=\int V_{F C}-\int \widehat{V}_{F C}$ is the FC voltage estimation error to be driven to zero by the adaptive estimator. Subtracting (8) from (10) and using (11) leads to,

$\dot{e}+K_{i} \int e+K_{p} e=\Psi^{\mathrm{T}} \tilde{\Theta}$

However, the regression vector $\Psi$ is unknown or uncertain since it contains the unknown parameters $\theta_{8}$ and $\theta_{9}$. Therefore, using the parameter estimates $\hat{\theta}_{8}$ and $\hat{\theta}_{9}$ makes only an approximation of $\widehat{\Psi}$ available for the online estimator. Thus, the error dynamic equation becomes,

$\dot{e}+K_{i} \int e+K_{p} e=\sigma$

where, $\sigma=\widehat{\Psi}^{T} \widehat{\Theta}-\Psi^{T} \Theta$. This can be written in a state-space form as,

$\dot{X}=A X+B U$

where, $X \in \mathbb{R}^{2}=\left[\int e_{b}, e_{b}\right]^{T}$ is the state vector and $U \in \mathbb{R}=\sigma$ is the state-space input. $A \in \mathbb{R}^{2 \times 2}$ is a stable matrix, and $B \in$ $\mathbb{R}^{2}$, are given by $A=\left[\begin{array}{cc}0 & 1 \\ -K_{i} & -K_{p}\end{array}\right]$ and $B=\left[\begin{array}{l}0 \\ 1\end{array}\right]$.

Henceforth, the estimator gains $K_{p}$ and $K_{i}$ may be chosen to place the closed-loop poles at their desired locations by using a pole placement technique or by solving the algebraic Riccati equation.

Theorem 1: Consider a nonlinear system in the form of (8) with the estimation law (10). The closed-loop system stability is achieved with the following adaptation law:

$\dot{\hat{\Theta}}=-\Gamma \widehat{\Psi} B^{T} P X$

where $\Gamma=\left[\gamma_{1}, \gamma_{2}, \ldots, \gamma_{9}\right]$ and $\gamma_{i}$ is a positive constant gain. $P$ is a symmetric positive definite matrix chosen to satisfy the following Lyapunov equation:

$A^{T} P+P A=-Q$ 
where $Q$ is a positive definite matrix.

Proof 1: Choose the following Lyapunov candidate:

$V=X^{T} P X+\tilde{\Theta}^{T} \Gamma^{-1} \tilde{\Theta}$

Taking the derivative of $V$ yields:

$\dot{V}=\dot{X}^{T} P X+X^{T} P \dot{X}+2 \tilde{\Theta}^{T} \Gamma^{-1} \dot{\hat{\Theta}}$

The FC parameters $\Theta$ are considered to be slowly time- varying so that $\dot{\tilde{\Theta}}=\dot{\hat{\Theta}}$. Substituting $\dot{X}$ from (14) yields,

$$
\dot{V}=[A X+B U]^{T} P X+X^{T} P[A X+B U]+2 \tilde{\Theta}^{T} \Gamma^{-1} \dot{\hat{\Theta}}
$$

Therefore, setting $U=\sigma$ implies that,

$\dot{V}=X^{T}\left[A^{T} P+P A\right] X+2 \sigma B^{T} P X+2 \widetilde{\Theta}^{T} \Gamma^{-1} \dot{\hat{\Theta}}$

Setting $A^{T} P+P A=-Q$ as in (16) leads to,

$\dot{V}=-X^{T} Q X+2 \sigma B^{T} P X+2 \widetilde{\Theta}^{T} \Gamma^{-1} \dot{\hat{\Theta}}$

Add and subtract $\widehat{\Psi}^{T} \Theta$ from $\sigma=\widehat{\Psi}^{T} \widehat{\Theta}-\Psi^{T} \Theta$ yields,

$\sigma=\widehat{\Psi}^{T} \Theta-\widehat{\Psi}^{T} \widehat{\Theta}+\Psi^{T} \Theta-\widehat{\Psi}^{T} \Theta$

Therefore,

$\sigma=\widetilde{\Psi}^{T} \tilde{\Theta}-\widetilde{\Psi}^{T} \Theta$

Substituting $\sigma$ in (21),

$\dot{V}=-X^{T} Q X+2 \tilde{\Theta}^{T}\left[\Psi^{T} \hat{\beta} B^{T} P X+\Gamma^{-1} \dot{\hat{\Theta}}\right]+2 \widetilde{\Psi}^{T} \Theta B^{T} P X$

Setting the adaptation law $\dot{\widehat{\Theta}}$ as defined in (15) implies that,

$\dot{V}=-X^{T} Q X+2 \widetilde{\Psi}^{T} \Theta B^{T} P X \leq-X^{T} Q X+\left|2 \widetilde{\Psi}^{T} \Theta B^{T} P X\right|$

With a proper selection of the control gains $K_{p}$ and $K_{i}$, it is possible to make the positive definite matrix $Q$ large enough so that the first term of (25), i.e., $-X^{T} Q X$, becomes dominant in a region where $X \neq 0$ [39]. Consequently, $\dot{V} \leq 0$ except possibly in the region of $X=0$. Therefore, the system is stable in the sense of Lyapunov. The region of $X=0$ is defined by the second term of (25), i.e., $\left|2 \widetilde{\Psi}^{T} \Theta B^{T} P X\right|$, which depends on the approximation uncertainty $\widetilde{\Psi}$ and gets smaller as $\widetilde{\Psi} \rightarrow 0$, i.e., $\tilde{\theta}_{8}, \tilde{\theta}_{9} \rightarrow 0$.

Another worth noting aspect is that persistent excitation (PE) condition is necessary in the adaptive parameter estimation. In a FC system, this condition can be satisfied due to the variation of the operating current which will eventually lead to a change in the output voltage. PE condition assures parameter convergence if the following condition in (26) is satisfied for all $\mathrm{t}_{0}$. It should be noted that $\alpha_{0}, \alpha_{1}$, and $\beta$ are all positive and $W$ is the regressor vector. The integral of $W W^{T}$ needs to be positive definite and bounded over all intervals of length $\beta$ which implies that $W$ must vary adequately over the interval $\beta$ to span the whole dimensional space.

$\alpha_{0} I_{n} \leq \int_{t_{0}}^{t_{0}+\beta} W W^{T} d t \leq \alpha_{1} I_{n}$

Algorithm 1 describes the iterative procedure of the proposed real-time NLAPE for extracting the PEMFC model parameters. From Algorithm 1, the proposed NLAPE has five vector multiplications, two scalar multiplications, one vector addition, two scalar additions/subtractions, and one vector transpose operation. The time complexity of a single application of the proposed method will be in the order of $O(n)$. To evaluate the performance of the proposed method, it is compared with EKF and $\mathrm{KF}$ in this work. However, the explanation of EKF and KF for identifying the parameters of the PEMFC model is considered superfluous as it is available in details in [30]. Despite the proposed method, EKF and KF perform several time-consuming arithmetic operations, as explained in [40, 41]. Utilizing the standard classical methods, the total time complexity of EKF and KF becomes $O\left(n^{3}\right)$, and employing faster algorithms, mentioned in [42], makes them $O\left(n^{2.372}\right)$.

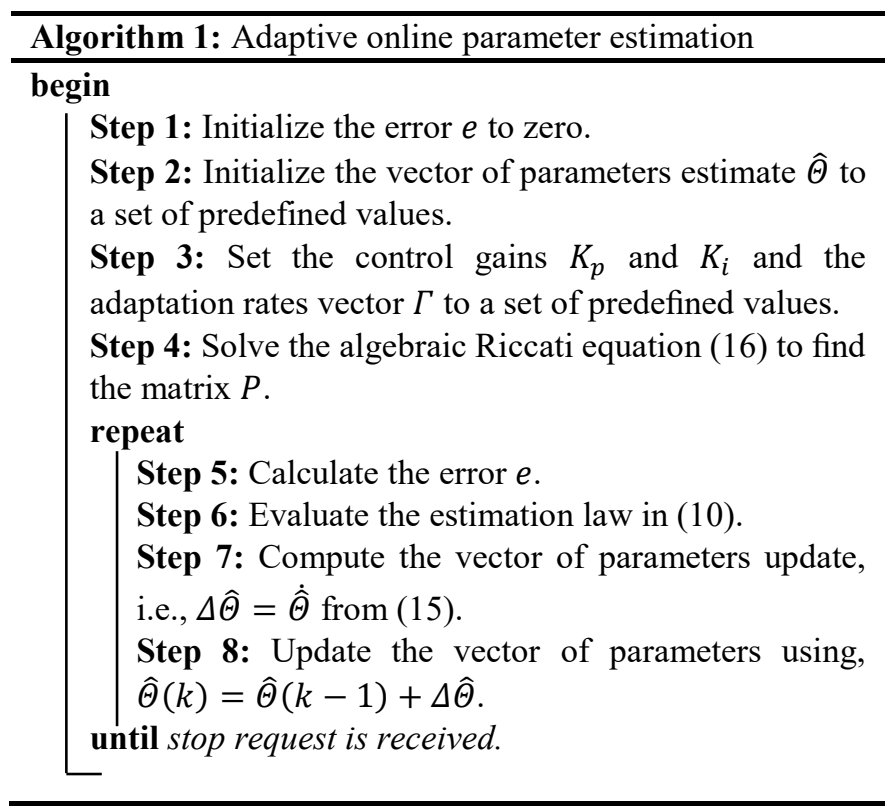

\section{EXPERIMENTAL SET-UP}

To corroborate the performance of the proposed NLAPE for the PEMFC parameters estimation, an experimental test rig has been developed. Fig. 3 illustrates this set-up where a Horizon $\mathrm{H}-500$ is employed as the main component regarding the estimation process. The technical characteristics of this PEMFC are reported in Table II. This PEMFC is air-cooled and has two axial fans on the stack housing to supply the oxygen of the cathode and remove the heat. It is also self-humidified and operates based on a dead-ended anode (DEA) principle in which the dry hydrogen is constantly supplied at a regulated inlet pressure. Therefore, there is a hydrogen supply valve in the anode inlet which provides dry hydrogen to the 


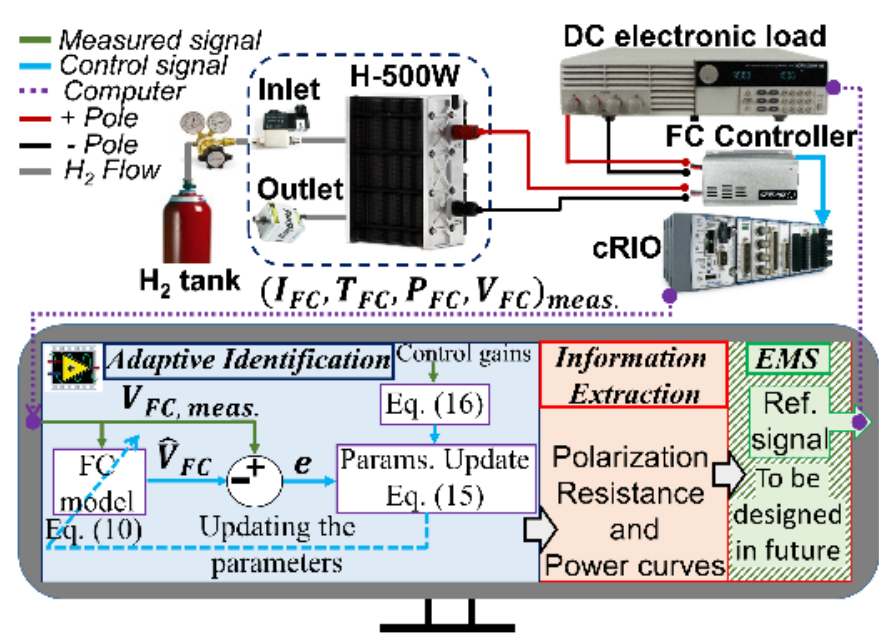

Fig. 3. The experimental set-up for testing the proposed online estimator.

PEMFC with a flow rate between 0 and $7 \mathrm{l} / \mathrm{min}$ according to the drawn current from the stack. The use of a DEA system has mitigated the need of a hydrogen recirculation loop (pump, water separator, and humidifier) in this PEMFC. Moreover, throughout the DEA operation, water back flow in the anode across the membrane results in self-humidification to a certain extent. On the other hand, the buildup of water in the catalyst and gas diffusion layers of anode and the nitrogen penetration from the cathode through the membrane have necessitated a regular purging to remove them. Consequently, the anode outlet is equipped with a hydrogen purging valve, which is normally closed. As advised by the manufacturer, a cyclic purging is performed every $10 \mathrm{~s}$ for a duration of $100 \mathrm{~ms}$ while the PEMFC is under operation to refill the anode volume with fresh hydrogen. The manual forward pressure regulator, shown in Fig. 3, retains the hydrogen partial pressure between 0.5 and 0.6 bar. During the purging of the accumulated water and nitrogen, the hydrogen flow depends on the difference between the atmosphere pressure and the anode side pressure. To limit the membrane damage, the pressure difference between the anode and cathode sides must not exceed 0.5 bar. The other hydrogen supply subsystems of this PEMFC are a mass flowmeter and a hydrogen tank. An OMEGA flowmeter (FMA-A2309), which is calibrated for hydrogen gas, is utilized to measure the flow. It is equipped with a capillary thermal technology to accurately determine mass flow devoid of any temperature, pressure, or square root corrections.

As shown in Fig. 3, the PEMFC is connected to a National Instrument CompactRIO (NI cRIO-9022) via its controller. The axial fan and the inlet/outlet valves operate using the controller

TABLE II

INTENDED PARAMETERS SPECIFICATIONS OF THE HORIZON H-500 PEMFC

\begin{tabular}{ll}
\hline \hline \multicolumn{2}{c}{ PEMFC technical data } \\
\hline Type & Open cathode \\
Number of cells & 36 \\
Hydrogen pressure & $0.5-0.6$ bar \\
Cathode pressure & $1 \mathrm{Bar}$ \\
Ambient temperature & 5 to $30^{\circ} \mathrm{C}$ \\
Maximum stack temperature & $65^{\circ} \mathrm{C}$ \\
Hydrogen purity & $99.999 \%$ dry $\mathrm{H}_{2}$ \\
Size & $130 \times 220 \times 122 \mathrm{~mm}$ \\
Cooling & Air (integrated cooling fan) \\
\hline \hline
\end{tabular}

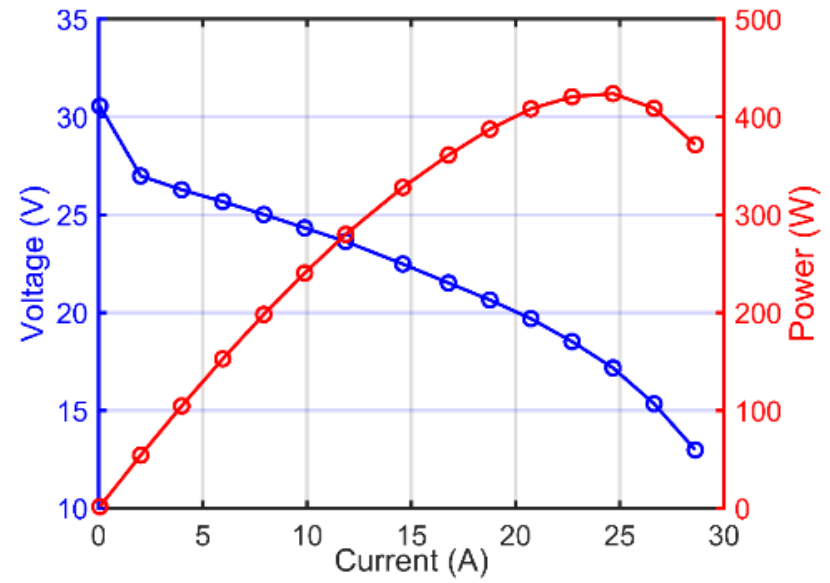

Fig. 4. The characteristics of the used PEMFC

of the PEMFC. The communication between CompactRIO and $\mathrm{PC}$ is done by an Ethernet connection. LabVIEW software is available in the PC. The data between the CompactRIO and the $\mathrm{PC}$ are transferred every $100 \mathrm{~ms}$. Current,

temperature, and voltage of the PEMFC stack are recorded for updating the model using the adaptive estimator. An 8514 BK Precision DC Electronic Load is employed to request a load profile from the PEMFC stack.

For the validation purpose of this work, a Horizon H-500 PEMFC which has been degraded through time is utilized. The exact age of this PEMFC is not known as it has been employed in different projects. In this respect, to clarify its present state of health, the polarization and power curves of this PEMFC are represented in Fig. 4. These curves are also used as a reference to evaluate the performance of characteristics estimation by the proposed adaptive estimator. To obtain the polarization curve of this PEMFC, a constant current has been applied to the PEMFC, and the corresponding output voltage and temperature have been recorded. By slightly increasing the current to different levels, the voltage and temperature of the stack can be recorded. After each increase of current, 15 to 25 minutes have been given to the PEMFC system to attain the stable condition. From Fig. 4, it is seen that the utilized PEMFC reaches the maximum power of almost $430 \mathrm{~W}$, and the maximum current seems to be around $30 \mathrm{~A}$ while for a brand-new H-500 PEMFC, it is $42 \mathrm{~A}$ as reported by the manufacturer. It should be noted that the conducted tests in this work have been performed in the ambient temperature of $20^{\circ} \mathrm{C}$ and humidity level of $60 \%$.

\section{RESUlTS AND DISCUSSION}

To evaluate the performance of the proposed NLAPE under different excitation signals, two current profiles have been imposed to the FC system. In the first place, its performance is compared with EKF, which is a well-known method in this line of work, to show its competence in extracting the PEMFC characteristics. Subsequently, it is compared with KF to clarify the importance of estimating maximum current in addition to the linear parameters.

The first load profile has been obtained from a city driving cycle (urban dynamometer driving schedule) using the IEEE VTS Motor Vehicles Challenge 2017 [43]. This profile can 
replicate the driving condition that a PEMFC may experience in a vehicular application. Fig. 5 represents the online estimate process concerning this load profile. Fig. 5a presents the drawn current from the PEMFC system and the corresponding stack temperature fluctuation. The estimation of the PEMFC voltage by the suggested NLAPE and EKF is shown in Fig. $5 \mathrm{~b}$ and Fig. $5 \mathrm{c}$. From these two figures, both parameter estimation methods can estimate the PEMFC voltage with a very good level of accuracy. To tangibly demonstrate the accuracy level of the voltage estimation, Fig. $5 \mathrm{~d}$ demonstrates the parity plot of the estimated stack voltage obtained by each of the estimators. In accordance with this figure, the estimated voltage points remain very close to the reference line and are dispersed all around it considering both methods. This parity plot validates the acceptable performance of the proposed nonlinear estimator in terms of voltage estimation and shows that it can reach the same level of precision as EKF while the deriving equations are much simpler in terms of mathematical operations. It is worth noting that the estimation mismatch around $30 \mathrm{~V}$ in Fig. 5d has happened in the very beginning of estimation process, which corresponds to almost the beginning of the requested current (Fig. 5a), and after that it disappears due to the convergence of
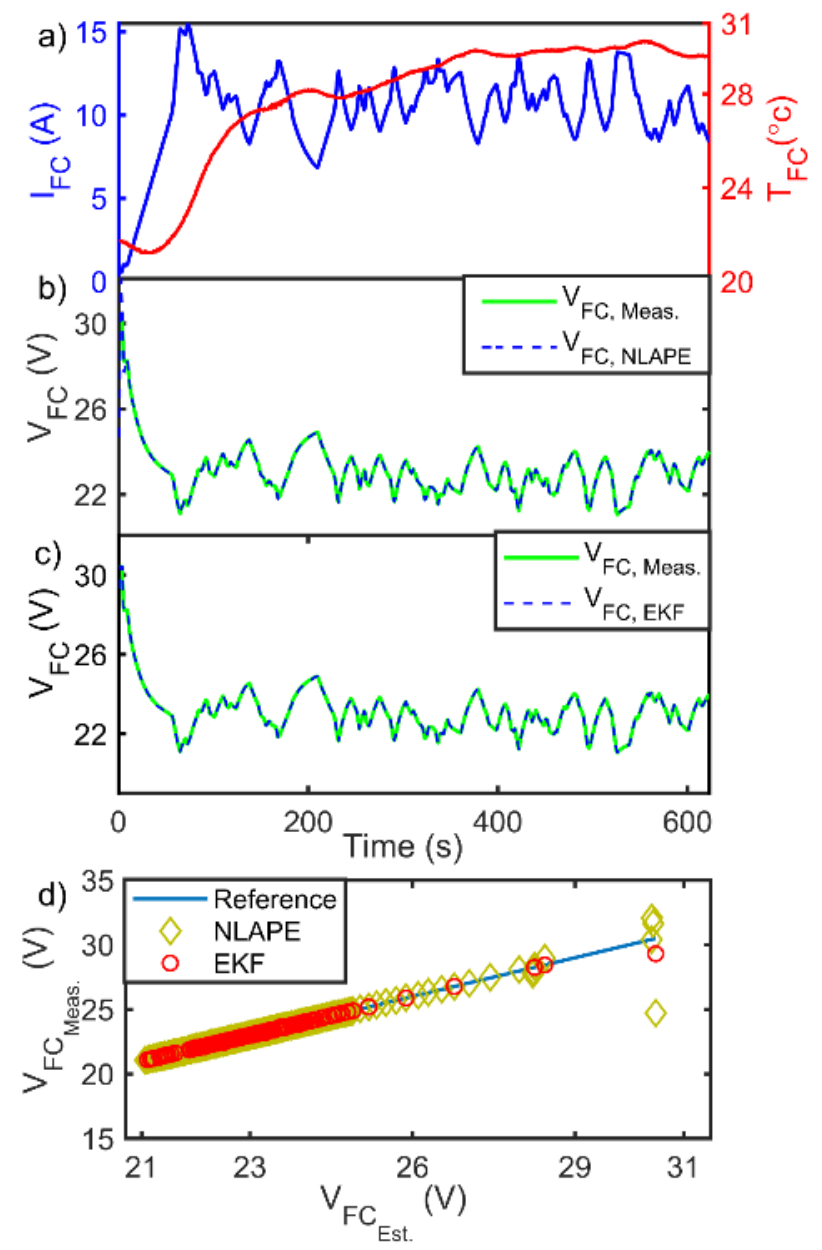

Fig. 5. Online estimation of PEMFC voltage using the first load profile, a) drawn current from the PEMFC stack (blue line) and the corresponding stack temperature (red line), b) voltage estimation by the proposed NLAPE, c) voltage estimation by EKF, and d) parity plot of the voltage estimation by both estimators. parameters. This is mainly due to the initialization of the tuning parameters, such as the control gains $K_{p}$ and $K_{i}$ and the adaptation rates vector $\Gamma$ in the proposed algorithm. The second profile utilized in the performance assessment of this work is a step-up current profile covering the whole operating range of the utilized FC. Fig. 6 illustrates the results of estimation for this second load profile. Fig. 6a shows the requested current and the variation of the stack temperature resulted from this load profile. It can be seen that temperature of the stack rises as the demanded current from the output voltage of the PEMFC stack. The comparison of the estimated voltage by the NLAPE and EKF is illustrated in Fig. 6d in form of a parity plot. It can be observed that a high density of the estimated points by both parameter identification methods is placed on the reference line which is obtained by plotting the measured PEMFC output voltage points. The estimation mismatch around $30 \mathrm{~V}$ in Fig. $6 \mathrm{~d}$ is due to the initialization of tuning parameters and swiftly disappears after the convergence of the parameters.

After discussing the performance of the identification methods regarding the voltage estimation, the next analysis concerns the estimation of polarization and power curves. Extraction of these curves is essential for an EMS design as the former can reflect
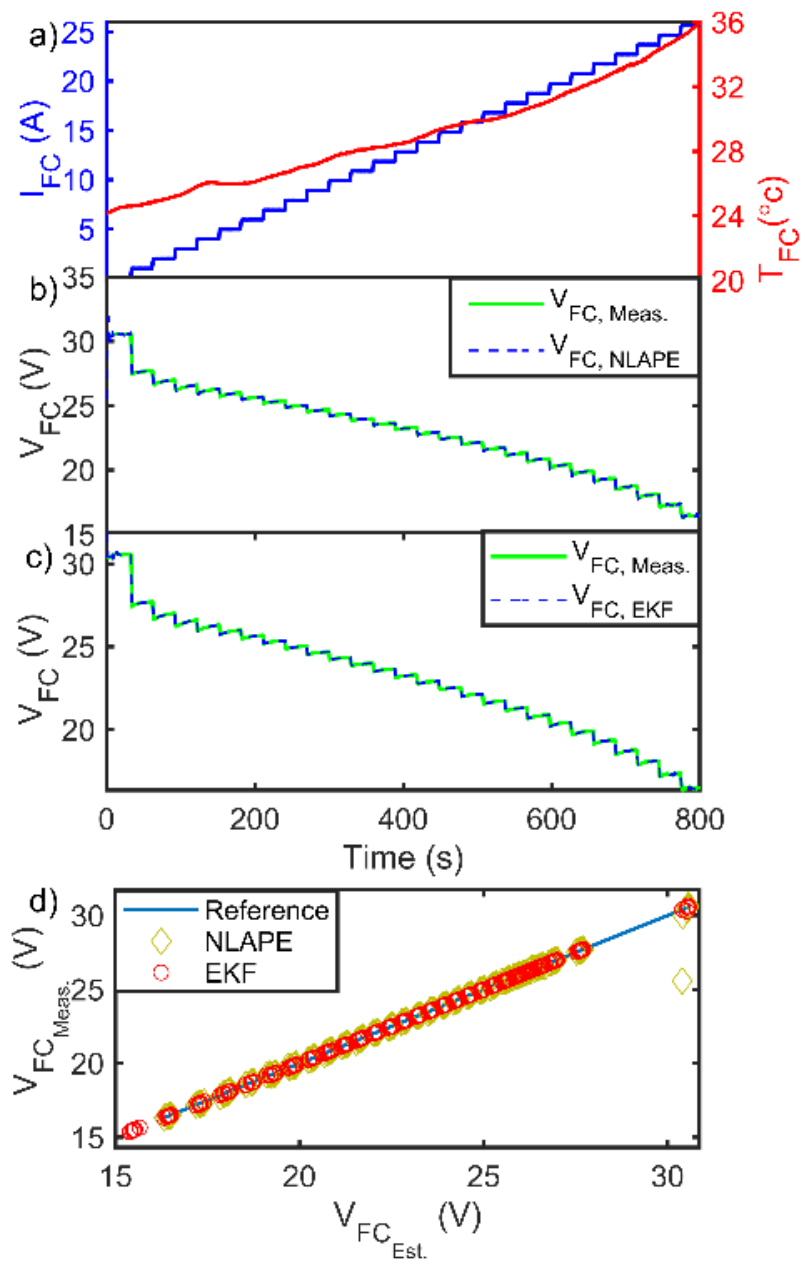

Fig. 6. Online estimation of PEMFC voltage using the second load profile, a) drawn current from the stack (blue line) and the corresponding temperature (red line), b) voltage estimation by NLAPE, c) voltage estimation by EKF, and d) parity plot of voltage estimation for both estimators. 
the health state and the latter can determine the MP. Another aspect which should be pointed out here is that the adaptive estimators are normally capable of tracking the output voltage precisely as they attempt to minimize the error of voltage estimation for one single point regardless of how they vary the parameters. However, it is important to check if this estimation is valid within the whole operating current range of the stack. Fig. 7 compares the estimation of these curves obtained by the proposed method, EKF, and KF. It should be noted that KF is included in the analysis in Fig. 7 mainly to highlight the influence of ignoring the maximum current estimation and consider it constant similar to most of the papers in the literature. Hence, the maximum current value is fixed at $42 \mathrm{~A}$ for $\mathrm{KF}$ since it is reported by the manufacturer for a brand-new PEMFC. However, for the case of NLAPE and EKF, the initial value is $42 \mathrm{~A}$, and they are supposed to estimate the actual value. Fig. 7 a presents the extracted polarization and power curves after $270 \mathrm{~s}$ from applying the first load profile. Fig. $7 \mathrm{~b}$ shows the extracted curves at $507 \mathrm{~s}$ while demanding the second current load profile from the PEMFC system. It should be noted these curves have been obtained from the PEMFC model using the estimated parameters at each specified timestep. At each case, the estimated curves are compared with the reference ones demonstrated in Fig. 4. To clarify the accuracy of characteristics extraction by the three estimators, residual standard error $(\mathrm{RSE})\left(R S E=\sqrt{\frac{\sum_{i=1}^{n}\left(y_{i}-\hat{y}_{i}\right)^{2}}{n-d}}\right.$, where $y_{i}$ is the
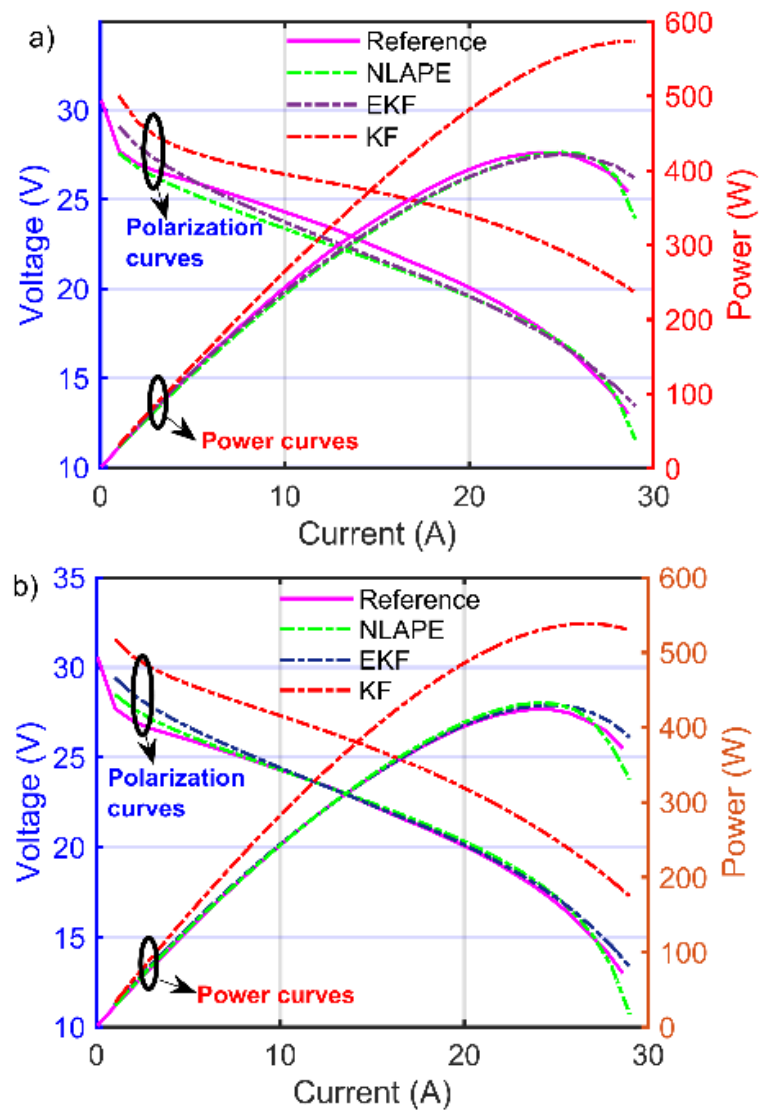

Fig. 7. Estimation of the PEMFC characteristics under the load profiles, a) extracted characteristics after applying the first load profile at $270 \mathrm{~s}, \mathrm{~b}$ ) extracted characteristics after applying the second load profile at $507 \mathrm{~s}$. observed value, $\hat{y}_{i}$ is the estimated value, $n$ is the total number of observations, and $d$ is the number of unknown parameters) is calculated for each of the considered case studies and reported in Table III. Furthermore, Table III compares the execution time of the proposed method with the other two estimators for each timestep. The reported value for each method is the average of execution time of ten consecutive timesteps. According to this table, NLAPE has been able to estimate the FC characteristics with a good level of accuracy compared to other two methods while its runtime is almost half of them. KF has shown the weakest performance in terms of RSE as it cannot tune the maximum current which is a nonlinear parameter.

Fig. 8 illustrates the estimation of the maximum current (nonlinear parameter) by both of NLAPE and EKF. As explained earlier, the initial value for maximum current is chosen as $42 \mathrm{~A}$. However, since the utilized PEMFC in this study is not new, this value has almost converged to $30 \mathrm{~A}$ which agrees with the maximum current value in the presented experimental polarization curve in Fig. 4. Therefore, 30 A has been selected as the reference value for this parameter. From Fig. 8, it can be seen that in case of the first profile, NLAPE and EKF converge to the reference value at almost $100 \mathrm{~s}$ and $200 \mathrm{~s}$ while in the second profile they both converge at around $350 \mathrm{~s}$. To check the required time for the utilized algorithms to estimate the PEMFC power characteristics, which is important for designing an EMS, the convergence trend of the maximum power for the first and second profile is presented in Fig. 9. To obtain this plot, the estimated power curve of the PEMFC has been plotted at each timestep and the maximum power value has been extracted from it. This trend shows the needed time to extract the targeted characteristics from the model, which is the main purpose of using these online estimators. The reference maximum power $(430 \mathrm{~W})$ has been extracted from the illustrated experimental power curve in Fig. 4. Since the two performed tests are not very long, this value is not expected to change due to degradation. From Fig. 9, it is observed that NLAPE converges to the reference value around $100 \mathrm{~s}$ sooner than EKF in the first profile. Regarding the second profile, they both almost converge to the reference value at $350 \mathrm{~s}$. Table IV summarizes the mean squared error (MSE) of the estimated

TABLE III

COMPARISON OF THE UTILIZED ESTIMATORS

\begin{tabular}{|c|c|c|c|}
\hline Estimator & $\begin{array}{l}\text { RSE (first } \\
\text { profile) }\end{array}$ & $\begin{array}{l}\text { RSE (second } \\
\text { profile) }\end{array}$ & $\begin{array}{l}\text { Average runtime of a } \\
\text { time step }(\mathrm{ms})\end{array}$ \\
\hline NLAPE & $\begin{array}{c}\text { Polarization: } \\
0.51 \\
\text { Power: } \\
109.56\end{array}$ & $\begin{array}{c}\text { Polarization: } \\
0.24 \\
\text { Power: } \\
58.93\end{array}$ & 4.5 \\
\hline $\mathrm{EKF}$ & $\begin{array}{c}\text { Polarization: } \\
0.58 \\
\text { Power: } \\
142.17\end{array}$ & $\begin{array}{c}\text { Polarization: } \\
0.53 \\
\text { Power: } \\
98.73\end{array}$ & 10.6 \\
\hline KF & $\begin{array}{c}\text { Polarization: } \\
20.27 \\
\text { Power: } \\
1.0543 \mathrm{e} 4\end{array}$ & $\begin{array}{c}\text { Polarization: } \\
15.85 \\
\text { Power: } \\
8.8581 \mathrm{e} 3 \\
\end{array}$ & 8.4 \\
\hline
\end{tabular}



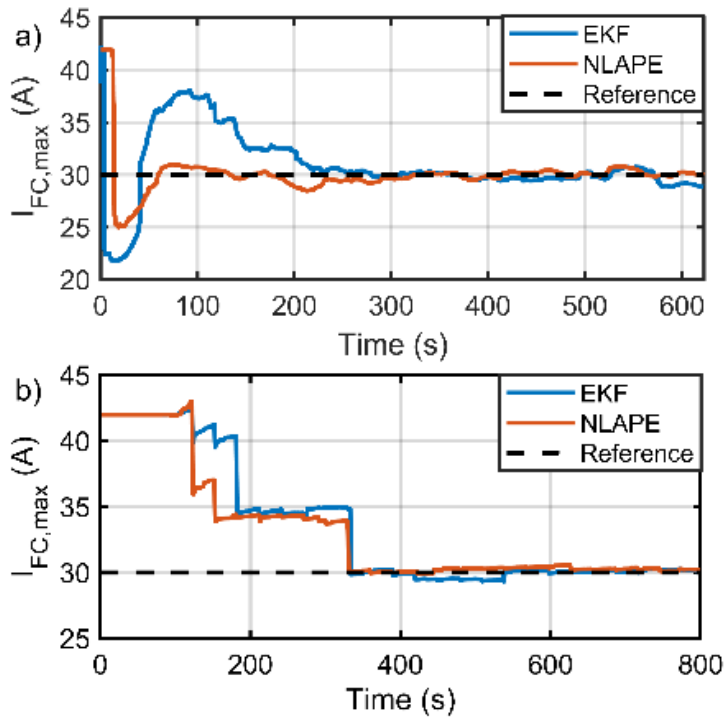

Fig. 8. Estimation of maximum current for the first current profile (a) and the second current profile (b).
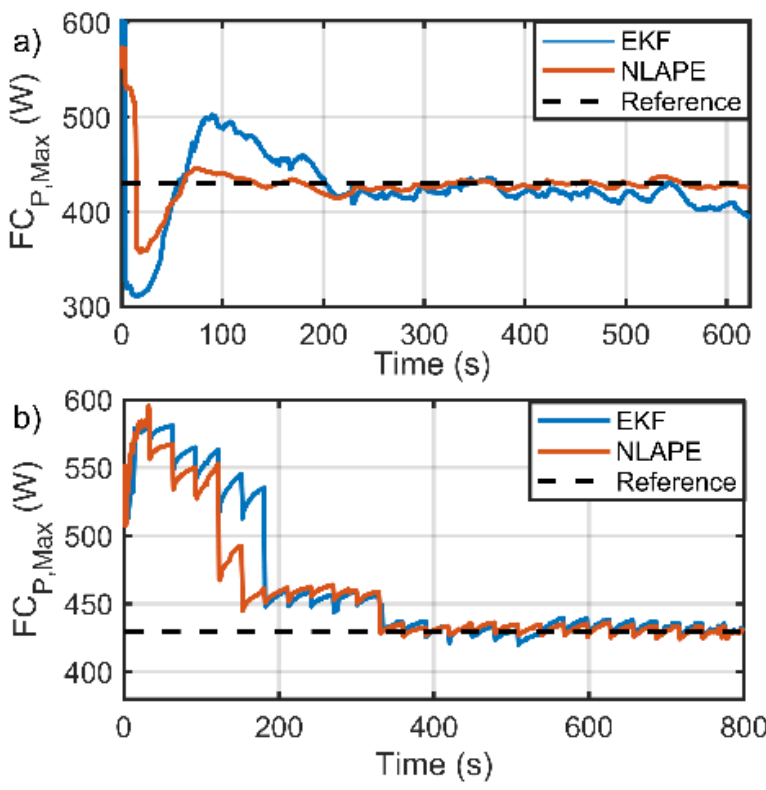

Fig. 9. Estimation of maximum power for the first current profile (a) and the second current profile (b).

maximum current and maximum power considering the explained reference values. This table shows that the proposed estimator has been capable of estimating both parameters with higher accuracy under the two current profiles.

TABLE IV

COMPARISON OF THE UTILIZED ESTIMATORS

\begin{tabular}{ccc}
\hline \hline Parameter Estimator & MSE (first profile) & MSE (Second profile) \\
\hline \multirow{3}{*}{ NLAPE } & Maximum current: & Maximum current: \\
& 4.15 & 27.74 \\
& Maximum power: & Maximum power: \\
& 477.2 & 2701.1 \\
& & \\
EKF & Maximum current: & Maximum current: \\
& 11.45 & 34.48 \\
& Maximum power: & Maximum power: \\
& 1437.2 & 3622.4 \\
\hline \hline
\end{tabular}

Another relevant analysis is related to the evolution of internal resistor which is obtained through estimating three parameters $R_{\text {internal }}=f\left(\zeta_{1}, \zeta_{2}, \zeta_{3}\right)$. Fig. 10a presents the estimated internal resistor for the case of first load profile and Fig. 10b shows the estimation result regarding the second profile. To validate the estimation evolution of the $\mathrm{FC}$ internal resistance, the result of a current interrupt test from [30] is utilized as a means of comparison. It should be noted that this test has been done on the same FC technology with similar conditions. Current interrupt is an offline characterization technique which is usually used to provide a rough estimate of the internal resistance at different operating conditions. Table V summarizes the results of the resistor measurement from the offline technique. Comparing the estimated values in Fig. 10 with the measured values in Table $\mathrm{V}$ shows that the estimated internal resistance range is consistent with the measured one.
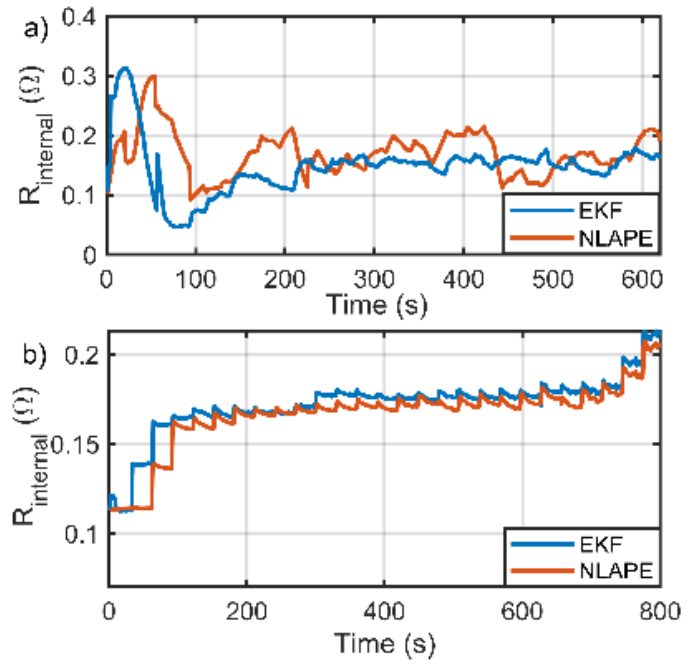

Fig. 10. Resistor estimation during the first load profile (a) and the second current profile (b).

TABLE V

MEASURED RESISTANCE BY CURRENT INTERRUPT

\begin{tabular}{ccc}
\hline \hline Current $(\mathrm{A})$ & Temperature $\left({ }^{\circ} \mathrm{C}\right)$ & Resistance $(\Omega)$ \\
\hline 3 & 24.4 & 0.1614 \\
6 & 24.8 & 0.1672 \\
9 & 26.4 & 0.1727 \\
12 & 27.3 & 0.1771 \\
15 & 28.8 & 0.1784 \\
18 & 31 & 0.1803 \\
21 & 33.2 & 0.1846 \\
24 & 36.8 & 0.1861 \\
26 & 39 & 0.2205 \\
\hline \hline
\end{tabular}

\section{CONCLUSION}

This paper puts forward a NLAPE for online health assessment of a PEMFC by tracing the required characteristics (power, voltage, resistance) for an EMS application. The PEMFC model is composed of nine parameters. All the parameters are linear-in-structure, except for one (maximum current), which is embedded in the nonlinear function. The proposed method capitalizes on the theory of adaptive control to identify online the explained linear and nonlinear parameters employing merely measured voltage, current, and temperature 
of the PEMFC stack. Firstly, it is compared with EKF, which is an established nonlinear estimator in the literature, to be validated in terms of accuracy for estimating the voltage and more importantly polarization and power curves. Secondly, the proposed NLAPE is compared with KF, which is a linear estimation approach, to highlight the importance of estimating maximum current (nonlinear parameter). As opposed to adaptive filtering and other gradient descent-based algorithms, the closed-loop stability of the utilized system is thoroughly studied by means of Lyapunov method. The competence of the proposed NLAPE is experimentally explored under two load profiles and compared with EKF and KF. The conducted comparison highlights the capability of the proposed approach in estimating the PEMFC output voltage and extracting the physical characteristics. The results of this paper suggest the following directions for future endeavors:

- The effectiveness of the proposed real-time parameter estimator can be validated by using several FCs with different aging milestones.

- The proposed estimator can be integrated into the design of an EMS to prevent the malfunction of the strategy while facing the FC performance drifts. To do so, the constraints and utilized parameters in the cost function of optimization-based EMSs can be updated using the proposed estimator. Moreover, the health state of the FC can be estimated and used in decisionmaking strategies.

\section{REFERENCES}

[1] İ. L. Sarioglu, O. P. Klein, H. Schroder, and F. Kucukay, "Energy Management for Fuel-Cell Hybrid Vehicles Based on Specific Fuel Consumption Due to Load Shifting," IEEE Transactions on Intelligent Transportation Systems, vol. 13, no. 4, pp. 1772-1781, 2012.

[2] J. Guanetti, S. Formentin, and S. M. Savaresi, "Energy Management System for an Electric Vehicle With a Rental Range Extender: A Least Costly Approach," IEEE Transactions on Intelligent Transportation Systems, vol. 17, no. 11, pp. 3022-3034, 2016.

[3] X. Tang, T. Jia, X. Hu, Y. Huang, Z. Deng, and H. Pu, "Naturalistic Data-Driven Predictive Energy Management for Plug-in Hybrid Electric Vehicles," IEEE Transactions on Transportation Electrification, pp. 1-1, 2020.

[4] Y. Huang, H. Wang, A. Khajepour, B. Li, J. Ji, K. Zhao, and C. Hu, "A review of power management strategies and component sizing methods for hybrid vehicles," Renewable and Sustainable Energy Reviews, vol. 96, pp. 132-144, 2018/11/01/ 2018.

[5] Y. Zhou, A. Ravey, and M.-C. Péra, "Real-time cost-minimization power-allocating strategy via model predictive control for fuel cell hybrid electric vehicles," Energy Conversion and Management, vol. 229, p. 113721, 2021/02/01/2021.

[6] K. Song, X. Wang, F. Li, M. Sorrentino, and B. Zheng, "Pontryagin's minimum principle-based real-time energy management strategy for fuel cell hybrid electric vehicle considering both fuel economy and power source durability," Energy, vol. 205, p. 118064, 2020/08/15/2020.

[7] Q. Li, T. Wang, C. Dai, W. Chen, and L. Ma, "Power Management Strategy Based on Adaptive Droop Control for a Fuel Cell-BatterySupercapacitor Hybrid Tramway," IEEE Transactions on Vehicular Technology, vol. 67, no. 7, pp. 5658-5670, 2018.

[8] J. Chen, C. Xu, C. Wu, and W. Xu, "Adaptive Fuzzy Logic Control of Fuel-Cell-Battery Hybrid Systems for Electric Vehicles," IEEE Transactions on Industrial Informatics, vol. 14, no. 1, pp. 292-300, 2018.

[9] M. Kandidayeni, A. M. Fernandez, L. Boulon, and S. Kelouwani, "Efficiency Upgrade of Hybrid Fuel Cell Vehicles' Energy
Management Strategies by Online Systemic Management of Fuel Cell," IEEE Transactions on Industrial Electronics, pp. 1-1, 2020.

[10] A. Forrai, H. Funato, Y. Yanagita, and Y. Kato, "Fuel-cell parameter estimation and diagnostics," IEEE Transactions on Energy Conversion, vol. 20, no. 3, pp. 668-675, 2005.

[11] X. Hu, C. Zou, X. Tang, T. Liu, and L. Hu, "Cost-Optimal Energy Management of Hybrid Electric Vehicles Using Fuel Cell/Battery Health-Aware Predictive Control," IEEE Transactions on Power Electronics, vol. 35, no. 1, pp. 382-392, 2020.

[12] M. Yue, S. Jemei, and N. Zerhouni, "Health-Conscious Energy Management for Fuel Cell Hybrid Electric Vehicles Based on Prognostics-Enabled Decision-Making," IEEE Transactions on Vehicular Technology, vol. 68, no. 12, pp. 11483-11491, 2019.

[13] K. Song, Y. Ding, X. Hu, H. Xu, Y. Wang, and J. Cao, "Degradation adaptive energy management strategy using fuel cell state-of-health for fuel economy improvement of hybrid electric vehicle," Applied Energy, vol. 285, p. 116413, 2021/03/01/ 2021.

[14] H. Li, Y. Zhou, H. Gualous, H. Chaoui, and L. Boulon, "Optimal Cost Minimization Strategy for Fuel Cell Hybrid Electric Vehicles Based on Decision-Making Framework," IEEE Transactions on Industrial Informatics, vol. 17, no. 4, pp. 2388-2399, 2021.

[15] D. Zhou, A. Al-Durra, I. Matraji, A. Ravey, and F. Gao, "Online Energy Management Strategy of Fuel Cell Hybrid Electric Vehicles: A Fractional-Order Extremum Seeking Method," IEEE Transactions on Industrial Electronics, vol. 65, no. 8, pp. 67876799, 2018

[16] P. T. Bankupalli, S. Ghosh, L. Kumar, S. Samanta, and S. Jain, "Operational Adaptability of PEM Fuel Cell for Optimal Voltage Regulation With Maximum Power Extraction," IEEE Transactions on Energy Conversion, vol. 35, no. 1, pp. 203-212, 2020.

[17] N. Bizon, "Energy optimization of fuel cell system by using global extremum seeking algorithm," Applied Energy, vol. 206, pp. 458474, 2017/11/15/2017.

[18] Q. Li, L. Yin, H. Yang, T. Wang, Y. Qiu, and W. Chen, "MultiObjective Optimization and Data-Driven Constraint Adaptive Predictive Control for Efficient and Stable Operation of PEMFC System," IEEE Transactions on Industrial Electronics, pp. 1-1, 2020.

[19] Q. Li, W. Yang, L. Yin, and W. Chen, "Real-Time Implementation of Maximum Net Power Strategy Based on Sliding Mode Variable Structure Control for Proton-Exchange Membrane Fuel Cell System," IEEE Transactions on Transportation Electrification, vol. 6, no. 1, pp. 288-297, 2020.

[20] M. Kandidayeni, A. M. F, L. Boulon, and S. Kelouwani, "Efficiency Enhancement of an Open Cathode Fuel Cell Through a Systemic Management," IEEE Transactions on Vehicular Technology, vol. 68, no. 12, pp. 11462-11472, 2019.

[21] A. M. Fernandez, M. Kandidayeni, L. Boulon, and H. Chaoui, "An Adaptive State Machine Based Energy Management Strategy for a Multi-Stack Fuel Cell Hybrid Electric Vehicle," IEEE Transactions on Vehicular Technology, vol. 69, no. 1, pp. 220-234, 2020.

[22] M. Kandidayeni, A. O. M. Fernandez, A. Khalatbarisoltani, L. Boulon, S. Kelouwani, and H. Chaoui, "An Online Energy Management Strategy for a Fuel Cell/Battery Vehicle Considering the Driving Pattern and Performance Drift Impacts," IEEE Transactions on Vehicular Technology, vol. 68, no. 12, pp. 11427$11438,2019$.

[23] T. Wang, Q. Li, Y. Qiu, L. Yin, L. Liu, and W. Chen, "Efficiency Extreme Point Tracking Strategy Based on FFRLS Online Identification for PEMFC System," IEEE Transactions on Energy Conversion, vol. 34, no. 2, pp. 952-963, 2019.

[24] T. Wang, Q. Li, L. Yin, and W. Chen, "Hydrogen consumption minimization method based on the online identification for multistack PEMFCs system," International Journal of Hydrogen Energy, vol. 44, no. 11, pp. 5074-5081, 2019/02/26/ 2019.

[25] Q. Li, T. Wang, S. Li, W. Chen, H. Liu, E. Breaz, and F. Gao, "Online extremum seeking-based optimized energy management strategy for hybrid electric tram considering fuel cell degradation," Applied Energy, vol. 285, p. 116505, 2021/03/01/ 2021.

[26] T. Wang, Q. Li, X. Wang, Y. Qiu, M. Liu, X. Meng, J. Li, and W. Chen, "An optimized energy management strategy for fuel cell hybrid power system based on maximum efficiency range identification," Journal of Power Sources, vol. 445, p. 227333, 2020/01/01/ 2020. 
[27] K. Ettihir, L. Boulon, M. Becherif, K. Agbossou, and H. S. Ramadan, "Online identification of semi-empirical model parameters for PEMFCs," International Journal of Hydrogen Energy, vol. 39, no. 36, pp. 21165-21176, 2014/12/12/ 2014.

[28] G. Squadrito, G. Maggio, E. Passalacqua, F. Lufrano, and A. Patti, "An empirical equation for polymer electrolyte fuel cell (PEFC) behaviour," Journal of Applied Electrochemistry, vol. 29, no. 12, pp. 1449-1455, 1999/12/01 1999.

[29] K. Ettihir, M. Higuita Cano, L. Boulon, and K. Agbossou, "Design of an adaptive EMS for fuel cell vehicles," International Journal of Hydrogen Energy, vol. 42, no. 2, pp. 1481-1489, 2017/01/12/ 2017.

[30] M. Kandidayeni, A. Macias, A. A. Amamou, L. Boulon, S. Kelouwani, and H. Chaoui, "Overview and benchmark analysis of fuel cell parameters estimation for energy management purposes," Journal of Power Sources, vol. 380, pp. 92-104, 2018/03/15/ 2018.

[31] J. C. Amphlett, "Performance Modeling of the Ballard Mark IV Solid Polymer Electrolyte Fuel Cell," Journal of The Electrochemical Society, vol. 142, no. 1, p. 9, 1995.

[32] R. F. Mann, J. C. Amphlett, M. A. I. Hooper, H. M. Jensen, B. A. Peppley, and P. R. Roberge, "Development and application of a generalised steady-state electrochemical model for a PEM fuel cell," Journal of Power Sources, vol. 86, no. 1, pp. 173-180, 2000/03/01/ 2000.

[33] Y. Xing, J. Na, and R. Costa-Castelló, "Real-Time Adaptive Parameter Estimation for a Polymer Electrolyte Membrane Fuel Cell," IEEE Transactions on Industrial Informatics, vol. 15, no. 11, pp. 6048-6057, 2019.

[34] J. M. Correa, F. A. Farret, V. A. Popov, and M. G. Simoes, "Sensitivity analysis of the modeling parameters used in Simulation of proton exchange membrane fuel cells," IEEE Transactions on Energy Conversion, vol. 20, no. 1, pp. 211-218, 2005.

[35] O. E. Turgut and M. T. Coban, "Optimal proton exchange membrane fuel cell modelling based on hybrid Teaching Learning Based Optimization - Differential Evolution algorithm," Ain Shams Engineering Journal, vol. 7, no. 1, pp. 347-360, 2016/03/01/ 2016.

[36] J. Cheng and G. Zhang, "Parameter fitting of PEMFC models based on adaptive differential evolution," International Journal of Electrical Power \& Energy Systems, vol. 62, pp. 189-198, 2014/11/01/ 2014.

[37] A. Saadi, M. Becherif, A. Aboubou, and M. Y. Ayad, "Comparison of proton exchange membrane fuel cell static models," Renewable Energy, vol. 56, pp. 64-71, 2013/08/01/ 2013.

[38] L. Lu, M. Ouyang, H. Huang, P. Pei, and F. Yang, "A semiempirical voltage degradation model for a low-pressure proton exchange membrane fuel cell stack under bus city driving cycles," Journal of Power Sources, vol. 164, no. 1, pp. 306-314, 2007/01/10/ 2007.

[39] H. Chaoui, M. Khayamy, and A. A. Aljarboua, "Adaptive Interval Type-2 Fuzzy Logic Control for PMSM Drives With a Modified Reference Frame," IEEE Transactions on Industrial Electronics, vol. 64, no. 5, pp. 3786-3797, 2017.

[40] V. Vaidehi and C. N. Krishnan, "Computational Complexity of the Kalman Tracking Algorithm," IETE Journal of Research, vol. 44, no. 3, pp. 125-134, 1998/05/01 1998.

[41] C. Montella. The Kalman Filter and Related Algorithms: A Literature Review [Online]. Available: https://www.researchgate.net/publication/236897001_The Kalman Filter_and_Related_Algorithms_A_Literature_Review/citations

[42] F. L. Gall, "Powers of tensors and fast matrix multiplication," presented at the Proceedings of the 39th International Symposium on Symbolic and Algebraic Computation, Kobe, Japan, 2014. Available: https://doi.org/10.1145/2608628.2608664

[43] C. Depature, S. Jemei, L. Boulon, A. Bouscayrol, N. Marx, S. Morando, and A. Castaings, "IEEE VTS Motor Vehicles Challenge 2017 - Energy Management of a Fuel Cell\&\#x002F;Battery Vehicle," in 2016 IEEE Vehicle Power and Propulsion Conference (VPPC), 2016, pp. 1-6.

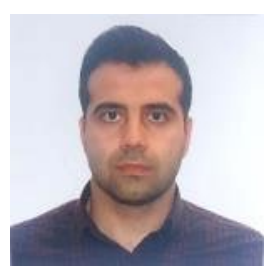

Mohsen Kandidayeni (S'18) was born in Tehran (Iran) in 1989. His educational journey has spanned through different paths. He received the B.S. degree in Mechanical Engineering in 2011, and then did a master's degree in Mechatronics at Arak University (Iran) in 2014. He joined the Hydrogen Research Institute of University of Quebec, Trois-Rivières (UQTR), QC, Canada, in 2016 and received his Ph.D. degree in Electrical Engineering from this university in 2020 . He is currently a postdoctoral researcher in electric-Transport, Energy Storage and Conversion Lab (e-TESC) at Université de Sherbrooke and a research assistant member in Hydrogen Research Institute of UQTR. He was a straight-A student during his Master and Ph.D. programs. Moreover, he has been the recipient of several awards/honors during his educational path, such as a doctoral scholarship from the Fonds de recherche du Québec-Nature et technologies (FRQNT), a postdoctoral scholarship from FRQNT, an excellence student grant from UQTR, and the $3^{\text {rd }}$ prize in Energy Research Challenge from the Quebec Ministry of Energy and Natural Resources. He has been actively involved in conducting research through authoring, coauthoring, and reviewing several papers in different prestigious scientific journals and also participating in various international conferences. His research interests include energy-related topics, such as hybrid electric vehicles, fuel cell systems, energy management, Multiphysics systems, modeling, and control.

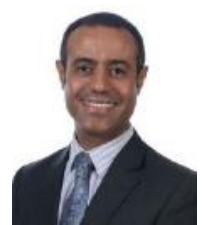

Hicham Chaoui (S'01-M'12-SM'13) received the Ph.D. degree in electrical engineering (with honors) from the University of Quebec, Trois-Rivières, QC, Canada, in 2011. His career has spanned both academia and industry in the field of control and energy systems. From 2007 to 2014, he held various engineering and management positions in the Canadian industry. He is currently an Associate Professor at Carleton University, Ottawa, ON, Canada and an Affiliate Professor at the University of Quebec, Trois-Rivières, QC, Canada. Prior to that, he was an Assistant Professor at Tennessee Technological University, TN, USA. His research interests include adaptive and nonlinear control theory, intelligent control, robotics, electric motor drives, and energy conversion and storage systems. His scholarly work has resulted in over 140 journal and conference publications. Dr. Chaoui is a senior member of IEEE and a registered professional engineer in the province of Ontario. He is also an Associate Editor of IEEE Transactions on Vehicular Technology and several other journals. Dr. Chaoui is a recipient of the Best Thesis Award and the Governor General of Canada Gold Medal Award. He is also a recipient of the Top Editor Award from IEEE Vehicular Technology Society.

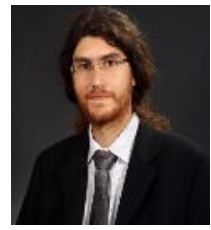

Loïc Boulon (M'10, SM'15) received the master's degree in electrical and automatic control engineering from the University of Lille (France), in 2006. Then, he obtained a $\mathrm{PhD}$ in electrical engineering from University of FrancheComté (France). Since 2010, he is a professor at UQTR (Full Professor since 2016) and he works into the Hydrogen Research Institute (Deputy director since 2019). His work deals with modeling, control and energy management of multiphysics systems. His research interests include hybrid electric vehicles, energy and power sources (fuel cell systems, batteries, and ultracapacitors). He has published more than 120 scientific papers in peer-reviewed international journals and international conferences and given over 35 invited conferences all over the word. In 2015, Loïc Boulon was general chair of the IEEE-Vehicular Power and Propulsion Conference in Montréal (QC, Canada). Prof. Loïc Boulon is now VP-Motor Vehicles of the IEEE Vehicular Technology Society and he found the "International Summer School on Energetic Efficiency of Connected Vehicles" and the "IEEE VTS Motor Vehicle Challenge". He is the holder of the Canada Research Chair in Energy Sources for the Vehicles of the future.

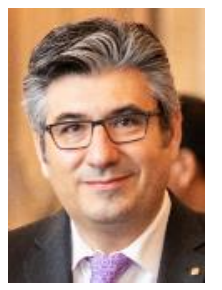

João Pedro F. Trovão (Senior Member, IEEE) received the M.Sc. degree and the Ph.D. degree in electrical engineering from the University of Coimbra, Coimbra, Portugal, in 2004 and 2013, respectively. From 2000 to 2014, he was a Teaching Assistant and an Assistant Professor with the Polytechnic Institute of CoimbraCoimbra Institute of Engineering (IPC- ISEC), Portugal. Since 2014, he has been a Professor with the Department of Electrical Engineering and Computer Engineering, University of Sherbrooke, Sherbrooke, QC, Canada, where he holds the 
Canadian Research Chair position in Efficient Electric Vehicles with Hybridized Energy Storage Systems. He is an Author/Co-Author of over 130 journal and conference papers. His research interests cover the areas of electric vehicles, hybridized energy storage systems, energy management and rotating electrical machines.

Dr. Trovão was General Chair of the 2018 IEEE Vehicle Power and Propulsion Conference, Chicago, IL, US. He was Technical Program Committee Co-Chair of the 2017 IEEE Vehicle Power and Propulsion Conference, Belfort, France, General Co-Chair and the Technical Program Committee Co-Chair of the 2014 IEEE Vehicle Power and Propulsion Conference, Coimbra, Portugal, as well as Award Committee Member for the 2015 (Montreal, Canada), 2016 (Hangzhou, China) and 2019 (Hanoi, Vietnam) IEEE Vehicle Power and Propulsion Conferences. He was a Guest Editor for the Special Issue of IET Electrical Systems in Transportation on Energy Storage and Electric Power Sub-Systems for Advanced Vehicles. He was a Guest Editor for the Special Issues of IEEE Transactions On Vehicular Technology On Electric Powertrains For Future Vehicles ANd On AdVAnced Vehicle Power Propulsion SYSTEMS. He is a Founding Member and the Director of the Electric-Transport, Energy Storage and Conversion (e-TESC) Lab of the University of Sherbrooke. $\mathrm{He}$ is a Senior Editor for the Automotive Electronics topic of the IEEE Vehicular Technology Magazine. 